\title{
The study of ultrasonic heat meter straight pipe length based on the CFD
}

\author{
WANG Wen-lin ${ }^{1}$, HUANGChao-chuan ${ }^{2}$, ZHANZhi-jie ${ }^{1}$ \\ 1.Zhejiang Institute of Metrology, Hangzhou 310018; 2.Zhejiang Cangnan Instrument Group Co., \\ Ltd, Cangnan 325802
}

Key words: ultrasonic heat meter; straight pipe; CFD

\begin{abstract}
In order to verifythe rationality of thestraight pipe'sdesign forthe ultrasonic heat meter calibration device, the Computational Fluid Dynamics(CFD) was used to the simulation for the DN25 heat meter, the straight pipe length of the domestic typical structure ultrasonic heat meter was determined through the analysis of the velocity distribution's difference in the straight pipe. It is gained that the straight pipe length before the heat meter is more than $14 \mathrm{D}$, the straight pipe length after the heat meter is more than $8 \mathrm{D}$ with the velocity distribution curve similarity in more than $99.5 \%$, and when multiple heat meters are connected in series, the last one is not affected by the previous one, the rationality of the design is confirmed, and it provides the theory basis for the design of the calibration device, and ensures the reliability of the measurement results.
\end{abstract}

\section{Introduction}

In the past ten years, the process of the heating commercialization in our country has developed rapidly, and the heat charge by household has become the development target of the heating, in the urban heating system reform, the heat meter, as the key equipment of the heat metering and charging, which is the key measurement apparatus of the trade transfer, has been listed in the national compulsory examination directory, and the first test is required for the installation[1]. However, the traditional standard device which based on the static mass method or the volume method is being used in the domestic various measurement technology institutions and production enterprises at present, although the verification accuracy is high, but there are many disadvantages, such as low efficiency, high energy consumption and so on, it has been difficult to adapt to the rapid development of heat meter industry. For this reason, the heat meter verificating device based On Flowrate-Time method with piston standard was designed in this topic, which used to the heat meter verification for the pipe size from DN15 to DN25. In the process of verification equipment design, the design of the straight pipe before and after the heat meter length have been become the key to the heat meter flow sensor measurement accuracy, if the design can not meet the requirements, the pipe will not be able to reach the state of the fully developed, the fluid flow velocity distribution of the deformation will occur or producing vortex, and caused large flow measurement error[2,3].

At present in most situations, the requirements of the straight pipe length in the process of the ultrasonic heat meter's installation widely used the industry standard of "the top ten and the after five" [4]. That the length of the straight pipe before an ultrasonic heat meter is at least 10D, the after length of the straight pipe is at least 5D, For this reason, the length of the straight pipe before an ultrasonic heat meter is $20 \mathrm{D}$, the after length of the straight pipe is at $10 \mathrm{D}$ are designed in the verification equipment, In order to verify the rationality of the design of straight pipe prover, the computational fluid dynamics (CFD) isusedto thenumerically simulation for theultrasonic heat meter, the reasonable length of straight pipe before and after is determined by the analysis ofthe change rule of fluid velocity distribution curve in the straight pipe before and after of the ultrasonic heat meter, and provide the corresponding theoretical basis to the verification equipment.

\section{The simulation model}

\subsection{Model buliding}

The simulation model is the ultrasonic heat meter on the market with the typical structure which used in the homes. According to the actual pipeline structure of calibration device, the structure 
model of the ultrasonic heat meter and the Straight pipe before and after are designed by the solidworks, as shown in figure 1. In this model, the round straight pipe is used in this experimental section, the length of the straight pipe before the ultrasonic heat meter for $20 \mathrm{D}$, after the length of the straight pipe is $15 \mathrm{D}$, the temperature sensor probe which inserted into the pipeline internal is installed in the bent pipe which in front of the ultrasonic heat meter. The ultrasonic heat meter used in the numerical simulation is DN25, the flow range is from $0.07 \mathrm{~m}^{3} / \mathrm{h}$ to $7 \mathrm{~m}^{3} / \mathrm{h}$, built-in "mirror" acoustic path arrangement is used in the model, the ultrasonic heat meter model is shown in figure 2.

In the pretreatment of CFD software Gambit to meshing simulation model, the tetrahedral grids are used in the model because of the existence of the straight pipe before the heat meter and heat meter for temperature sensor probe and the "mirror", and in order to obtain better simulation results, the gard is local cypher in the temperature sensor probe and the "mirror", as is shown in the figure 3, the straight pipe after the heat meter is cylinder, the Shape relative rules, and the hexahedron mesh is used in the pipe[5].

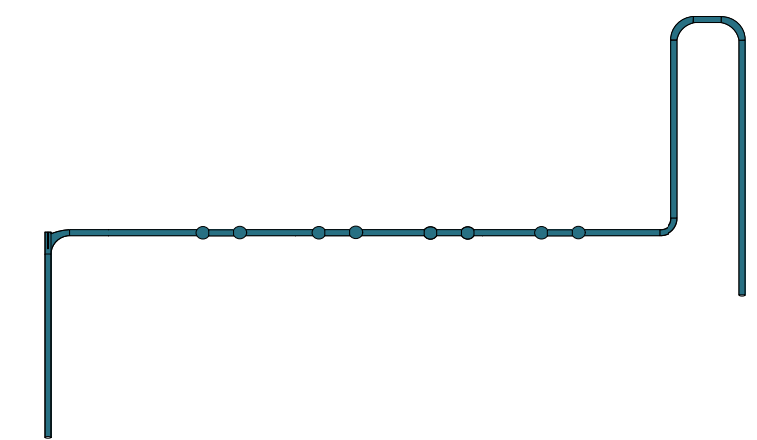

Fig. 1 The pipe model of the heat meter

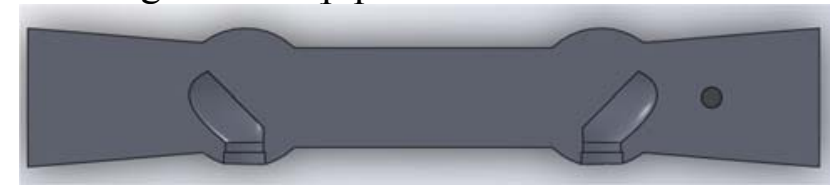

Fig. 2The model of the heat meter

\subsection{Parameter Settings}

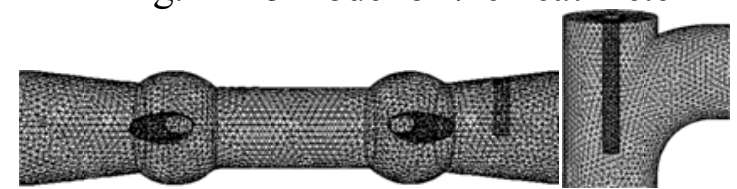

Fig. 3 The grid tumble figure

The three dimensional single precision solver is used in this model, the fluid media is the water in the $50^{\circ} \mathrm{C}$, the density is $988.03 \mathrm{~kg} / \mathrm{m}^{3}$, the inlet boundary is set with the velocity inlet, the outlet boundary is set with the out flow[6,7], according to the flow range of the heat meter, the maximum velocity in the inlet is $3.2 \mathrm{~m} / \mathrm{s}$.

The $k$-epsilon model in the turbulence model is used because of the reynolds number in the pipeline which less than 2320[8]. Besides setting inlet velocity, the turbulence intensity and the hydraulic diameter are used in the pipe to represent the other two turbulence parameters[9,10]. The hydraulic diameter is the diameter $\mathrm{D}$ of the pipe, the calculation of the turbulence intensity is shown in formula 1:

$$
I=0.16(\mathrm{Re})^{-\frac{1}{8}}=0.16\left(\frac{\rho v D}{\eta}\right)^{-\frac{1}{8}}
$$

In the formula, $I$ is the turbulence intensity, Re is the Reynolds number, $\rho$ is the density of the flow, $\mathrm{kg} / \mathrm{m}^{3}, v$ is the velocity of the flow, $\mathrm{m} / \mathrm{s}, \mathrm{D}$ is the the diameter of the pipe, $\mathrm{m}, \quad \eta$ is the dynamic viscosity of the flow, $\mathrm{Pa} \cdot \mathrm{s}$. 


\section{Numerical analysis}

After completion of the parameter Settings and start to iterative calculation of simulation model, the inlet velocityof theimulation modelis $3.2 \mathrm{~m} / \mathrm{s}$, for example, the number of iterations is less than 1000 times to achieve effective convergence, the speed of the pipe section cross section cloud picture as shown in figure 4.

In the figure 4 , figure (a) (f) is the monitoring surface speed cloud figure in theformer straight pipe of the ultrasonic heat meter which apart from the temperature sensor probe in $2 \mathrm{D}, 5 \mathrm{D}, 8 \mathrm{D}, 11 \mathrm{D}$, $14 \mathrm{D}$ and 15D. Due to the influence of the temperature sensor probe and the bend, after the bending, the velocity of the flow in the lower part of the pipe is significantly higher than that of the upper, the lower fluid flow velocity in the middle is less than two side, and then increased with the increasing of straight pipe length gradually tends to be stable, the velocity cloud figrue in $15 \mathrm{D}$ is similar to the 14D.

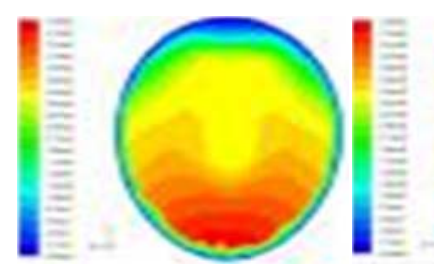

(a)

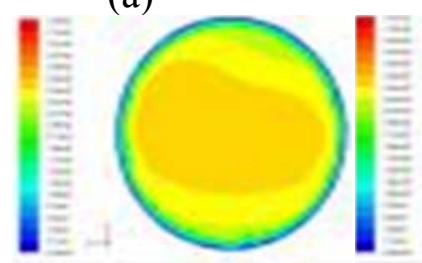

(c)

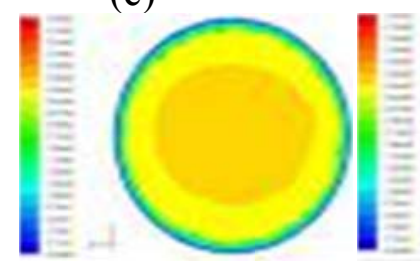

(e)

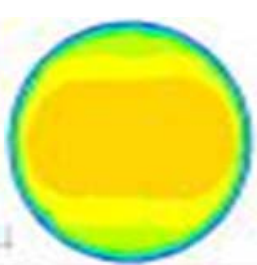

(b)

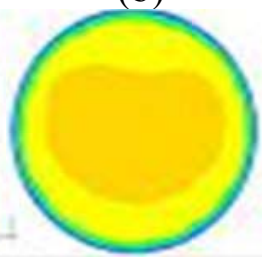

(d)

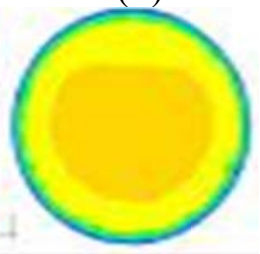

(f)

Fig. 4 The velocity profile in Monitoring surface

In order to accurately determine the length of straight pipe which reaches a steady state, the introduction of the similarity to determine whether the fluid reach a steady state, the calculation formula of such as shown in formula 2and formula 3 below:

$$
\begin{aligned}
& d(x, y)=\sqrt{\frac{\sum\left(x_{i}-y_{i}\right)^{2}}{n}} \\
& \operatorname{sim}(x, y)=\frac{1}{1+d(x, y)}
\end{aligned}
$$

In this formula, $\mathrm{x}$ and $\mathrm{y}$ are the monitoring velocity value in two pipe cross-section, $\operatorname{sim}(\mathrm{x}, \mathrm{y})$ is the similarity degree of the two sections.

The monitors are set in the $\mathrm{Y}$ axis and $\mathrm{Z}$ axis, the velocity distribution in the cross section of the Ultrasonic heat meter straight pipeis got, as is shown in the figure 5 and figure 6 . 


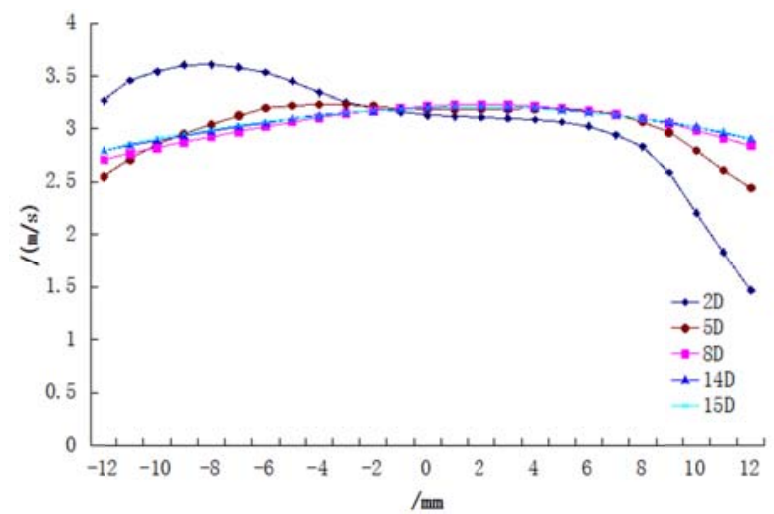

Fig. 5 The velocity profile in Y axis

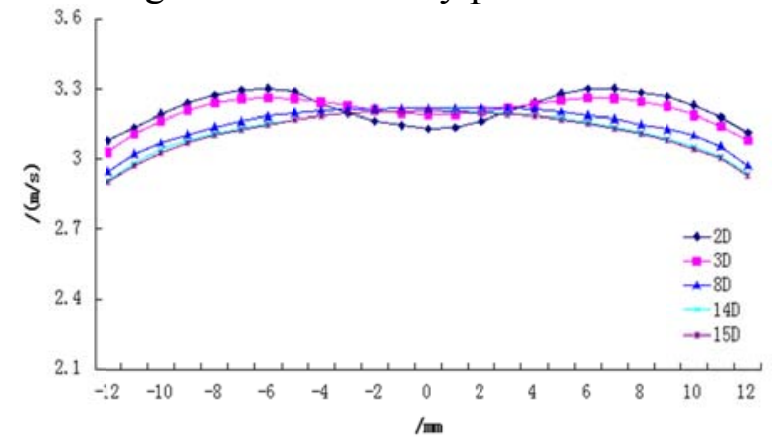

Fig. 6 The velocity profile in $\mathrm{Z}$ axis

The velocity at the bottom of the pipe is significantly higher than that of the upper in the 2D, when compared with the $2 \mathrm{D}$, the velocity in $8 \mathrm{D}$ increases in the upper part of the pipe, and the velocity decreases in the bottom part of the pipe, the velocity distribution curve in 14D and 15D have been achieve a sense of stability. It is gained that thelevel of similarity of 14D and 15D in the $\mathrm{Y}$ axis and $\mathrm{Z}$ axis are all above $99.5 \%$, it can be identified that the flow with 14D have been reached full development state, namely in the former straight pipe inlet velocity of $3.2 \mathrm{~m} / \mathrm{s}$, the length of the straight pipe before the DN25 ultrasonic heat meter for at least 14D.

Also the straight pipe after ultrasonic heat meter is also used the same method, get the speed of ultrasonic heat meter straight pipe section after section distribution curve, as is shown in the figure 7 and figure 8.

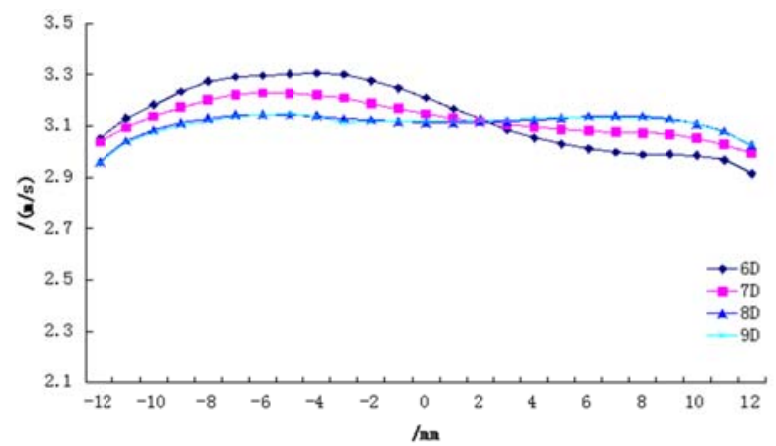

Fig. 7 The velocity profile in Y axis

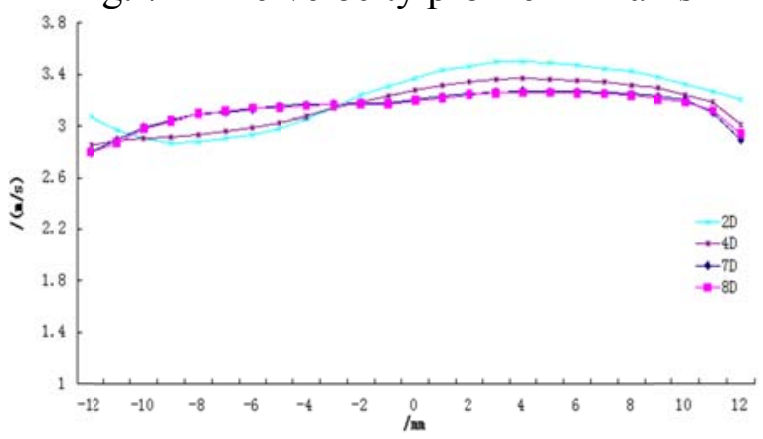

Fig. 8 The velocity profile in $\mathrm{Z}$ axis

In the $\mathrm{Y}$ axis, due to the influence of the temperature sensor probe and the bend, the velocity of 
the flow in the bottom part of the pipe is significantly higher than that of the upper, With the increase of ratio of straight pipe length, the velocity increases in the upper part of the pipe, and the velocity decreases in the bottom part of the pipe, and the velocity distribution curve in $8 \mathrm{D}$ and 9D have been achieve a sense of stability. In the $Z$ axis, the pipe on the right side of the speed of the fluid should be greater than the speed of the left side of the fluid, with the increase of ratio of straight pipe length, on the left side of the fluid flow rate increases, while the right side of the flow velocity of fluid is reduced, the stability was achieved to 7D and $8 \mathrm{D}$. It is gained that thelevel of similarity of $8 \mathrm{D}$ and $9 \mathrm{D}$ in the $\mathrm{Y}$ axis and $\mathrm{Z}$ axis are all above $99.5 \%$, it can be identified that the flow with $8 \mathrm{D}$ have been reached full development state, namely in the former straight pipe inlet velocity of $3.2 \mathrm{~m} / \mathrm{s}$, the length of the straight pipe after the DN25 ultrasonic heat meter for at least 8D.

With the different size of the Straight pipe before the inlet velocity, the length of the straight pipe are different, the ratio of straight pipe before and after the heat meter length and the relationship between the inlet velocity as shown in figure 9 .

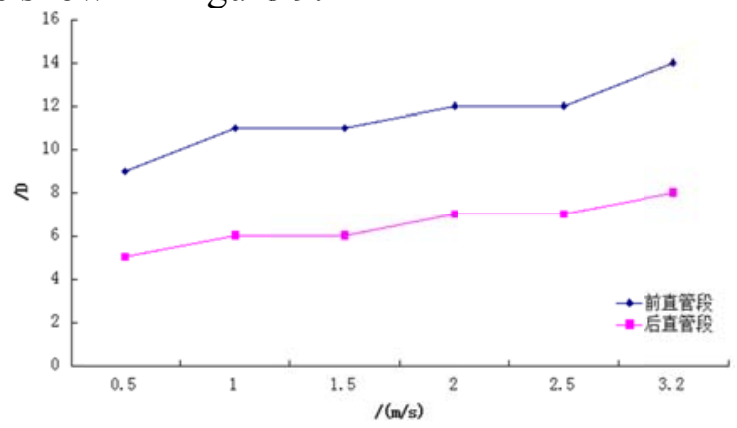

Fig.9straight section length and the inlet velocity diagram

In the figure 9, with the increase of the inlet velocity, the length of the pipe is increasing, it is gained that the length of the straight pipe before the DN25 ultrasonic heat meter for at least 14D, and the length of the straight pipe after the DN25 ultrasonic heat meter for at least 8D.

When multiple table series, in the same way to get the second straight pipe before and after ultrasonic heat meter only part of the cross section of the velocity distribution curve, as shown in figure 10, as shown in figure 11 and figure 12 and figure 13, found by calculation, when more than meter series, after the meter will not requirement for straight pipe before a meter.

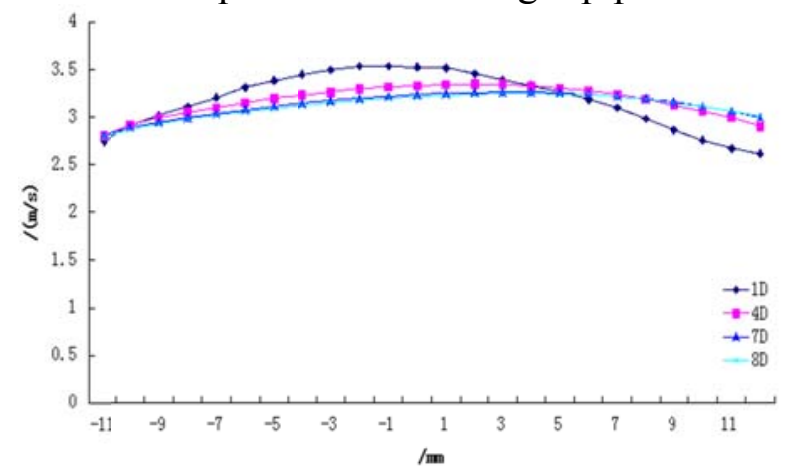

Fig.10The velocity profile in $\mathrm{Y}$ axis of the before

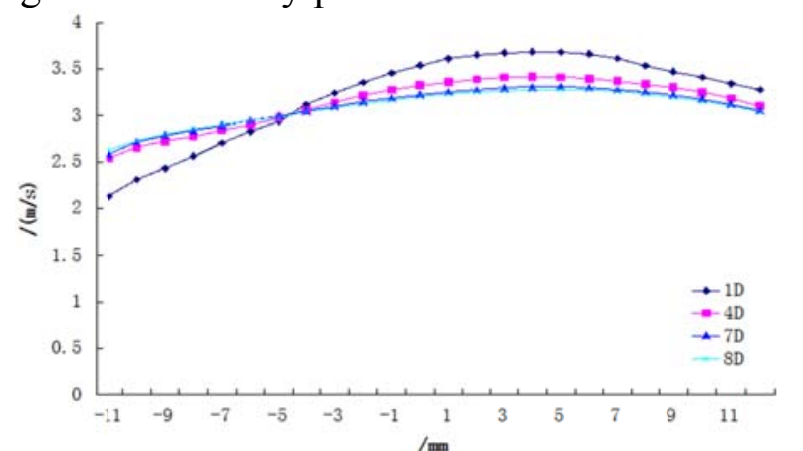

Fig. 11The velocity profile in $\mathrm{Z}$ axis of the before 


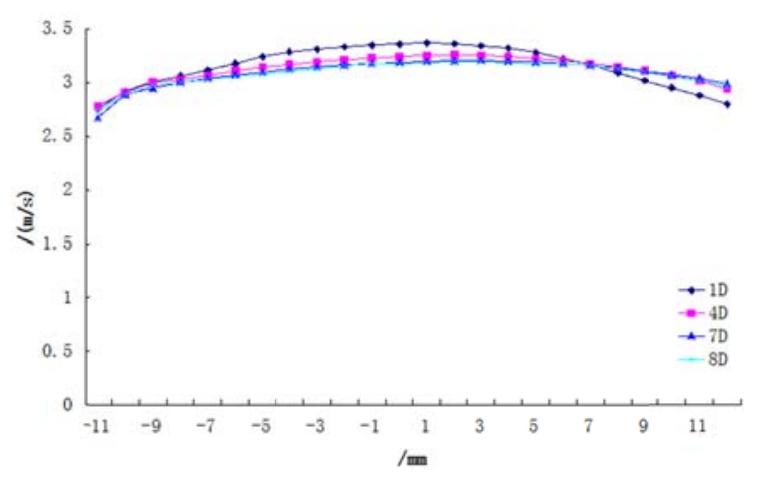

Fig. 12The velocity profile in $\mathrm{Y}$ axis of the after

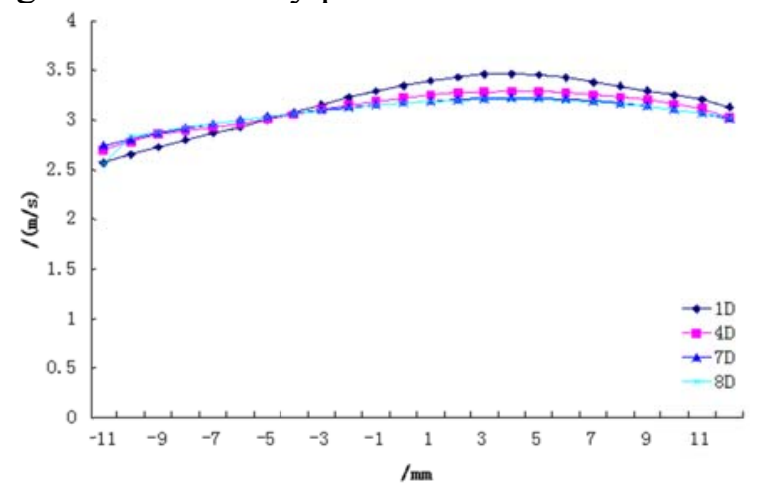

Fig. 13The velocity profile in $\mathrm{Z}$ axis of the after

\section{Conclusion}

The model of the DN25 ultrasonic heat meter is designed by the solidworks, the simulation for the model is done by the CFD, and it is gained that the length of the straight pipe before the DN25 ultrasonic heat meter for at least 14D, and the length of the straight pipe after the DN25 ultrasonic heat meter for at least $8 \mathrm{D}$. when multiple table series, after the meter will not requirement for straight pipe before a meter.

\section{Reference}

[1] LIU Zhi-hui. The Piston Type Verification Device for Heat Meters[D]. China JiLiang University, 2013:1-3.

[2] XIE Lin, YIN Jia-yun, JI Bo-feng,et al. Discuss on Suitable length for Straight Pipe of Flowmeter[J]. Petrochemical industry automation, 2014, 50(5): 58-62.

[3] LIANG Li-xin, MAI Wei-ming. The Error Variation Ultrasonic Flowmeter Affected by the Length straight[J]. Measurement and testing technology, 2001, 4: 12.

[4] GUO Xiao-li, ZHENG Dan-dan, ZHANG Tao, et al. Adaptability study of ultrasonic flowmeter in the flow field of reducer and straight pipe[J]. Sensor and micro system, 2012, 31(5): 68-72.

[5] Jun-jie zhou, guo-quan $\mathrm{xu}$, hua-jun zhang etc. Fluent software engineering technology and instance analysis [M]. Beijing: China water conservancy and hydropower press, 2010:39-44.

[6] Mr Lin, cheng-xi li, ren-xi hu, et al. Proficient in FLUENT6.3 flow field analysis [M]. Beijing: chemical industry press, 2009:47-56.

[7] Jiang fan, rats. Fluent advanced application and instance analysis [M]. Beijing: tsinghua university press, 2008:11-16.

[8] Su Yanxun guo-wei liang, ChengJian, etc. Flow measurement and testing [M]. China metrology press, 2007:17-21. 
[9] ZHU Wei-jun. Research on thermal gas flowmeter based on metallic-coated fiber Bragg gratings[D]. China JiLiang University, 2014:38-40.

[10] $X$ T Zhang, $Q$ M Mao, Z G Nie, et al. A Study of Composite Flow Meter Based on the Theory of Electromagnetic and Ultrasonic[C]//Applied Mechanics and Materials. 2014, 568: 309-314. 